\title{
RESULTS OF TESTS MADE WITH PLACEMENT FERTILI- ZATION ON THE KOTKANIEMI EXPERIMENTAL FARM
}

\author{
Yrjö Pessi, Mikko Ylänen, Auvo Leskelä and Jorma Syvälahti
}

Rikkihappo Oy, Helsinki

Received February 5, 1970

Various tests have been made with the placement of fertilizers in spring cereals in the 1960 's. On the one hand, the investigations aimed at finding out the kind of effect this method had on the crop yield, on the other hand, the technique of placement aroused interest. The results have shown that the increases in crop yields were considerable (ELONen et al. 1967, Elonen 1967, Heinonen \& Nieminen 1961, Köyljürrvi 1969, LaRpes 1966, 1968, Nieminen 1963, Nieminen et. al. 1967, Pessi 1967), expecially on clay soils. The best depth of placement seems to be 8-12 cm (NIEMinen et al. 1967). Placement also had its effect on the growth of cereals (Aura 1967, Elonen 1967, Elonen et al.1967), on the equality of ripening (KöYLIJ̈̈RVI, 1969), and on the quality of the crop (ELONEN 1967, Elonen et al. 1967, LARPEs 1966, Nieminen et al. 1967).

The tests were started on the Kotkaniemi Experimental Farm at Vihti in 1966. The technique of the tests can partly be seen from the headings of the Tables, but some details on the working technique are given below.

The fertilizers were placed at a depth of $8-10 \mathrm{~cm}$. The machine used in the placement was a Juko fertilizer-cultivator. On the plots on which the fertilizers were being broadcast the mixing into the soil was made with a rigid-tine harrow. As different preparation of soils might have affected the final results, all the same phases of work were carried out on the test plots, e.g. the broadcast plots were driven with an empty fertilizer-cultivator. Rotation tests were carried out to enable exact fertilization in addition to which the fertilizer amounts to each plot were checked by weighing.

All the tests were accompanied by effective weed control. Chlormequat (CCC) was in most cases used in connection with weed control sprayings. The plots were harvested with a combine-harvester.

The monthly mean temperatures and monthly precipitations measured at a place 10 kilometres from the experimental farm are presented in the Table 1. 
Table 1. Meteorological observations at Vihti during the growing seasons 1965-69.

Monthly precipitations in millimetres

\begin{tabular}{lrrrrrr} 
& \multicolumn{1}{c}{ V } & VI & VII & VIII & IX & Total \\
1965 & 8.3 & 18.1 & 106.9 & 90.0 & 65.0 & 288.3 \\
1966 & 15.1 & 40.7 & 74.9 & 28.3 & 77.7 & 236.7 \\
1967 & 51.9 & 21.8 & 27.8 & 142.1 & 53.3 & 296.9 \\
1968 & 70.5 & 30.6 & 67.1 & 111.4 & 74.4 & 354.0 \\
1969 & 21.6 & 15.5 & 45.3 & 40.1 & 95.9 & 218.4 \\
\hline Average & 33.5 & 25.3 & 64.4 & 82.4 & 73.3 & 278.8
\end{tabular}

Average temperatures per month in ${ }^{\circ} \mathrm{C}$

\begin{tabular}{lrrrrrc} 
& V & VI & VII & VIII & IX & $\begin{array}{c}\text { Average } \\
\text { (V-IX) }\end{array}$ \\
1965 & 7.7 & 16.0 & 14.6 & 13.4 & 11.6 & 12.66 \\
1966 & 9.4 & 17.9 & 17.9 & 13.8 & 7.9 & 13.38 \\
1967 & 10.1 & 14.7 & 17.6 & 15.2 & 10.5 & 13.62 \\
1968 & 7.4 & 16.2 & 14.5 & 15.3 & 10.0 & 12.68 \\
1969 & 8.7 & 15.3 & 16.2 & 15.2 & 9.6 & 13.00 \\
\hline Average & 8.7 & 16.0 & 16.2 & 14.6 & 9.9 &
\end{tabular}

Results (Tables 2-3, p. 195-196, and tables 4-6, p. 198-202)

Table 2 shows the average grain yields of two year tests in which the effect of the placement of different nutrients was studied. The placement of nitrogen has the greatest effect, even the placement of phosphorus and potassium seems to be of importance. Giving fertilizers together with seed also gave good results. In this case the moisture content of the crop was high at the moment of harvesting. Delay in ripening was noted throughout the growing time.

Results in Table 3 throw light on the importance of the placement of phosphorus. They show that although the basic fertilization with phosphorus was rather abundant, a smaller amount of phosphorus placed separately gave increases in crop yield $95 \%$ statistical reliability was not achieved, however.

Table 4 shows how the placement of NPK-amounts of different sizes affected the crop. The placement of the smallest amount of fertilizer gave an average increase of about 600 $\mathrm{kg} / \mathrm{ha}$. The biggest amount gave an increase of $400 \mathrm{~kg}$. The hectolitre-weight and the 1000-grain-weight rose simultaneously.

As seen in Table 2, the placement of nitrogen proved to be the most important. Tests were accordingly arranged during four years in order to compare nitrochalk $(26 \% \mathrm{~N})$ and urea. In the tests, fertilizers and the seed were sown with separate machines in parallel rows,

Tables 5 and 6 show that the increase in crop yield caused by urea was not as good as that caused by nitrochalk. The average value of the tests in Table 5 shows a difference of $380 \mathrm{~kg}$ per hectare, in Table 6 this difference is $100 \mathrm{~kg}$. Attention has to be paid to the annual fluctuation in the difference of crop yields. 
Table 2. Results of two trials in placement of nutrients in 1966-67 at Kotkaniemi.

Test in 1966: Soil type sandy muddy clay. Fertilizers: nitrochalk, superphosphate, and potassium chloride. Nutrient amounts correspond to $450 \mathrm{~kg} / \mathrm{ha}$ of compound fertilizer $15-20-15\left(\mathrm{~N}-\mathrm{P}_{2} \mathrm{O}_{5}-\mathrm{K}_{2} \mathrm{O}\right)$ which amount was used when all the nutrients were given simultaneously. Svenno-wheat sown 21. 5. 66. Size of test plot $5 \times 50 \mathrm{~m}$, harvested plot $3.6 \times 50 \mathrm{~m}$. 4 replications.

Test in 1967: Soil type silty sandy clay. Soil analysis in 1967: pH 6.0, Ca 3000, P 2.6 and K $170 \mathrm{mg} / \mathrm{l}$. The same fertilizers as in 1966, nutrient amounts correspond to $700 \mathrm{~kg} / \mathrm{ha}$ of fertilizer $15-20-15$. Svennowheat sown 16. 5. 67. Size of test plot $5 \times 66 \mathrm{~m}$, harvested plot $3.6 \times 66 \mathrm{~m}$. 3 replications.

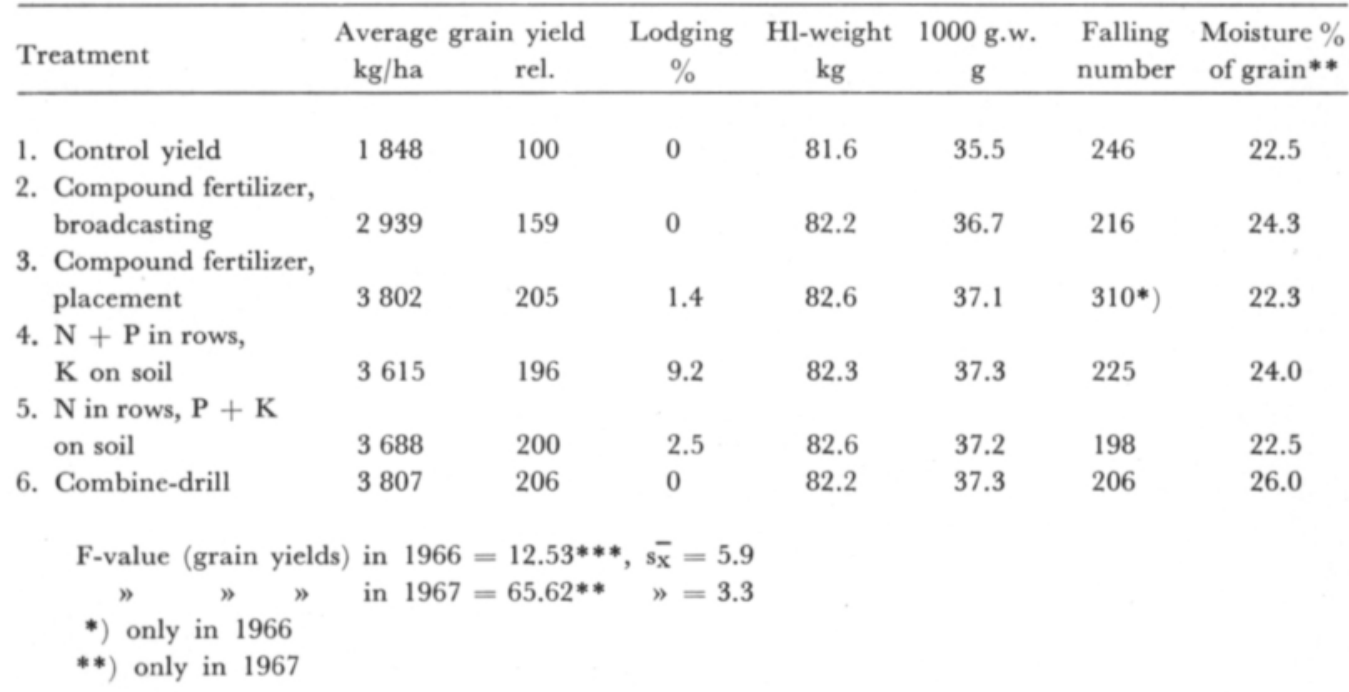

Baking test analysis (1966)

\begin{tabular}{|c|c|c|c|c|c|c|c|c|c|}
\hline Treatment & $\begin{array}{c}\text { Flour } \\
\text { yield } \\
\%\end{array}$ & $\begin{array}{l}\text { Swelling } \\
\text { number }\end{array}$ & $\begin{array}{l}\text { Gluten } \\
\% \text { SM }\end{array}$ & $\begin{array}{c}\text { Ash } \\
\%\end{array}$ & $\begin{array}{l}\text { Falling } \\
\text { number } \\
\text { of flour }\end{array}$ & $\begin{array}{c}\text { Raw } \\
\text { protein } \\
\%\end{array}$ & Dough & $\begin{array}{c}\text { Germi- } \\
\text { nated } \\
\text { grains } \\
\%\end{array}$ & $\begin{array}{c}\text { Green } \\
\text { grains } \\
\%\end{array}$ \\
\hline 1 & 67.9 & 16.0 & 33.0 & 0.62 & 199 & 13.6 & Good & 4.0 & - \\
\hline 2 & 63.4 & 15.0 & 39.0 & 0.67 & 197 & 16.1 & Good & 4.8 & - \\
\hline 3 & 65.5 & 14.0 & 38.0 & 0.62 & 196 & 14.5 & Good & 4.1 & 0.1 \\
\hline 4 & 67.8 & 12.0 & 37.0 & 0.66 & 208 & 14.8 & Good & 2.8 & 0.2 \\
\hline 5 & 66.6 & 14.0 & 39.0 & 0.59 & 213 & 15.8 & Good & 3.6 & 0.1 \\
\hline 6 & 65.1 & 14.0 & 40.0 & 0.62 & 189 & 15.8 & Good & 2.8 & 0.1 \\
\hline
\end{tabular}

In the tests described in Tables 5 and 6 , the fertilizer was placed with a separate machine after the seed was sown. Owing to this, the urea came to be at different distances from the seed row and it is evident that in some cases it came so near the seed row that it may have had a detrimental effect on the development of the cereals. 
Table 3. So-called start phosphorus in placement fertilization in 1966-1968 at Kotkaniemi.

In 1966: Soil type sandy muddy clay. Fertilization in autumn 1965: $800 \mathrm{~kg} / \mathrm{ha}$ of PK-fertilizer $(0-16.5-$ 16.5). Test made with Svenno-wheat. Size of test plot $5 \times 50 \mathrm{~m}$. Harvested plot $1.8 \times 50 \mathrm{~m}$. 2 replications. Start phosphorus given in powdered superphosphate.

In 1967: Soil type clayey silt and fine sand. Soil analysis in 1967: pH 5.9, Ca 1600, P 18 and K 208 mg/l. Fertilization in autumn: $1000 \mathrm{~kg} / \mathrm{ha}$ of PK-fertilizer $(0-16.5-16.5)$. Test made with Pendek-oats. Size of test plot $5 \times 30 \mathrm{~m}$. Harvested plot $3 \times 30 \mathrm{~m}$. 3 replications. Start phosphorus given in granular superphosphate.

In 1968: Soil type clayey humus soil. Soil analysis in 1967: pH 5.2, Ca 2700, P 2.9 and K 295 mg/l. Fertization under spring preparation of seed-bed: $800 \mathrm{~kg}$ of PK-fertilizer $(0-16.5-16.5)$ 31. 5. 68. Preceding crop fallow. Test made with Balder-barley. Sown 1.6. Size of test plot $5 \times 50 \mathrm{~m}$. Harvested plot $3 \times 50 \mathrm{~m}$. 4 replications. Start phosphorus given in granular superphosphate.

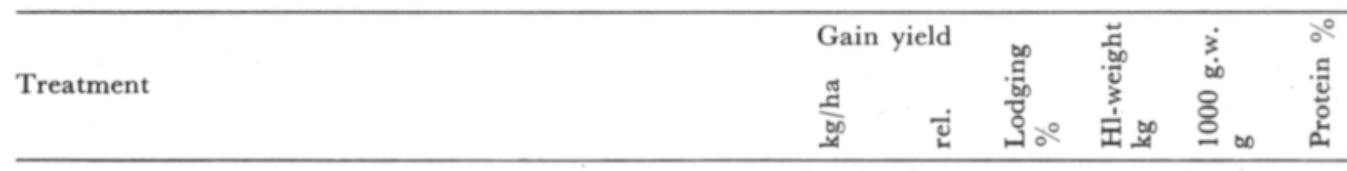

\section{In 1966 Svenno-wheat}

1. Basic fertilization in autumn $800 \mathrm{~kg} / \mathrm{ha}$ of PK-fertilizer $\quad \begin{array}{llllllll}1632 & 100 & 0 & 76.7 & 36.9\end{array}$

2. In spring additional $300 \mathrm{~kg} / \mathrm{ha}$ of Nitrochalk, placement $\quad \begin{array}{lllllll}3052 & 187 & 0 & 77.8 & 37.6 & -\end{array}$

3. Basic fertilization, Nitrochalk and in addition $100 \mathrm{~kg} / \mathrm{ha}$ of superphosphate

$\begin{array}{llllll}3174 & 194 & 0 & 77.8 & 36.7 \quad-\end{array}$ F-value of grain yields $=178.3 * *$, $\mathbf{s}_{\overline{\mathbf{x}}}=2.4 \%$

In 1967 Pendek-oats

1. Basic fertilization in autumn $1000 \mathrm{~kg} / \mathrm{ha}$ of $\mathrm{PK}$-fertilizer $\quad \begin{array}{lllllll}1675 & 100 & 0 & 55.4 & 32.5 & -\end{array}$

2. In addition $400 \mathrm{~kg} / \mathrm{ha}$ of Nitrochalk, placement $\quad \begin{array}{lllllll}4103 & 245 & 0 & 52.3 & 31.1\end{array}$

3. Basic fertilization, Nitrochalk and in addition $100 \mathrm{~kg} / \mathrm{ha}$ of superphosphate

$\begin{array}{llllll}4489 & 268 & 0 & 52.6 & 31.4 & -\end{array}$

F-value of grain yields $=33.53 * *$,

$\mathbf{s}_{\mathbf{x}}=7.7 \%$

\section{In 1968 Balder-barley}

1. Basic fertilization in spring $800 \mathrm{~kg} / \mathrm{ha}$ of $\mathrm{PK}$-fertilizer, broadcasting

$\begin{array}{llllll}3333 & 100 & 5.5 & 69.8 & 38.9 & 10.2\end{array}$

2. In addition $300 \mathrm{~kg} / \mathrm{ha}$ of Nitrochalk, placement

$\begin{array}{llllll}4267 & 128 & 77.5 & 65.6 & 28.4 & 11.4\end{array}$

3. Basic fertilization. Nitrochalk and in addition $100 \mathrm{~kg} / \mathrm{ha}$ of superphosphate

$$
\begin{aligned}
& \begin{array}{llllll}
4568 & 137 & 79.5 & 66.2 & 21.8 & 12.0
\end{array} \\
& \text { F-value of grain yields }=17.08^{* *} \text {, } \\
& \mathbf{s}_{\mathbf{x}}=3.9 \%
\end{aligned}
$$

Fertilization with start phosphorus has increased the grain"yields every year but this cannot be considered statistically reliable. 


\title{
Conclusions
}

On the basis of the results of the tests made on clay soils and on soils containing clay the following conclusions can be drawn:

Among different nutrients the placement of nitrogen proved to be the most important.

Placement of nutrients in rows at a depth of about 8-10 cm gave considerable increases in crop yield compared with ordinary broadcasting.

In the two tests where fertilizer and seed were sown in the same row, the crop yield was of the same size as in the case where they were sown separately. Ripening was delayed, however.

When fertilizer and seed were sown with separate machines and the fertilizer was placed closer than $10 \mathrm{~cm}$ to the seed, urea did not prove as good as nitrochalk.

\section{REFERENCES}

AurA, E. 1967. Effect of the placement of fertilizer on the development of spring wheat. J. Sci. Agric. Soc. Finl. 39: 148-155.

Elonen, P. 1967. Sprinkler irritation on day soils in southern Finland. Ibid. 39: 67-98.

- - Nieminen, L. \& KarA, O. 1967. Tuloksia kevätviljojen sadetuksesta savimailla. Maat. ja Koetoim. 21: 7-17.

Heinonen, R. \& Nieminen, L. 1961. Väkilannoitteiden rivikylvö. Maat. ja Koetoim. 15: 7-13.

HonkavaAra, T. 1950. Rakeistamisen ja rivikylvön vaikutuksesta fosforilannoitteiden tehoon. Maat. ja Koetoim. 4: 124-134.

KöyLIJÄRVI, J. 1969. Rivilannoitus kevätviljasatojen varmentajana. Pellervo 1969: 204-207.

LARPES, G. 1966. Rivilannoituksen vaikutus kevätviljoissa. Maat. ja Koetoim. 20: 14-20.

-n-1968. Rivilannoituskokeet vv. 1965-67. Koetoim. ja Käyt. n:o 4.

NiEMinen, L. 1963. Kannattaako sijoituslannoitus. Leipä leveämmäksi, n:o 4: 6-8.

Nieminen, L., Kara, O. \& Elonen, P. 1967. Kokemuksia sijoituslannoituksesta. Maat. ja Koetoim. 21: $42-49$.

Pessi, Y. 1967. Fertilizer placement in Finland. ISMA Joint techn. agric. Conf. Stresa 1967.

\section{SELOSTUS}

\section{RIVILANNOITUSKOKEIDEN TULOKSIA KOTKANIEMEN KOETILALLA}

\author{
Yrjö Pessi, Mikko Ylänen, Auvo Leskelä ja Jorma Syvälahti
}

Rikkihappo Oy, Helsinki

Kirjoituksessa selostetaan Vihdissä sijaitsevalla Kotkaniemen koetilalla vuosina 1966-1969 tehtyjen rivilannoituskokeiden tuloksia. Kokeet on tehty hiesupitoisilla savimailla. Tulosten perusteella voidaan tehdä mm. seuraavia päätelmiä:

Eri ravinteista on typen sijoitus osoittautunut tärkeimmäksi.

Ravinteiden sijoittaminen maahan riviin noin $8-10 \mathrm{~cm}: \mathrm{n}$ syvyyteen on antanut huomattavia sadonlisäyksiä tavanomaiseen hajalannoitukseen verrattuna.

Kahdessa kokeessa mukana ollut menetelmä, missä lannoite ja siemen on kylvetty samaan riviin, on antanut samansuuruisen sadon kuin kylvö erikseen. Tuleentuminen on kuitenkin viivästynyt.

Menetelmässä, missä lannoite ja siemen on kylvetty eri koneella ja missä lannoite voi joutua myös lähelle siementä, urea ei ole osoittautunut oulunsalpietarin veroiseksi. 
Table 4. Tests with placement fertilization 1967-1968 at Kotkaniemi

Soil type silty clay. Soil analysis in 1967: pH 5.8, Ca 2930, P 4.1 and K $170 \mathrm{mg} / \mathrm{l}$. Size of test plot $10 \times 30 \mathrm{~m}$, harvested plot $9 \times 30 \mathrm{~m} .4$ replications.

Treatment

\section{Paavo-barley 1968}

1. Control yield

764

100

64.50

62.0

33.9

$\begin{array}{ll}9.7 & 40.8\end{array}$

4.1

2. $450 \mathrm{~kg} / \mathrm{ha}$ of fertilizer $15-20-15$

$\begin{array}{rrrrrrrr}229 & 79.0 & 0 & 61.6 & 34.3 & 9.4 & 50.6 & 4.0 \\ 344 & 88.0 & 0 & 63.6 & 36.0 & 9.5 & 57.7 & 4.4 \\ 309 & 81.5 & 0 & 60.5 & 32.7 & 10.8 & 55.5 & 4.5 \\ 384 & 80.0 & 0 & 61.6 & 34.7 & 10.4 & 63.2 & 4.7\end{array}$

3. " "

$\begin{array}{cr}\left(\mathrm{N}-\mathrm{P}_{2} \mathrm{O}_{5}-\mathrm{K}_{2} \mathrm{O}\right) \text { broadcasting } & 1752 \\ & 2630\end{array}$

4. $900 \mathrm{~kg} / \mathrm{ha}$

broadcasting 2361

5. " "

placement

2931

F-value of grain yields $=21.51 * * *, s_{\bar{x}}=8.4 \%$

\section{Otra-barley 1968}

1.

2. See above

3. " "

4.

5.

\section{Otra-barley 1969}

1.

2. See above

3. " "

4.

5.

Pendek-oats 1968

1.

2. See above Paavo-barley

3. " "

4. " " "

5.

Svenno-wheat 1967

1.

2. See-above Paavo-barley

3. " " "

4.

5.

\begin{tabular}{lllllllll}
1128 & 100 & 77.8 & 0 & 64.1 & 37.3 & 10.6 & 52.5 & 4.1 \\
2142 & 190 & 85.8 & 0 & 64.0 & 38.4 & 10.3 & 66.6 & 4.4 \\
3112 & 276 & 91.3 & 0 & 65.4 & 39.6 & 10.1 & 72.0 & 4.4 \\
2671 & 237 & 84.0 & 4 & 61.2 & 36.9 & 12.2 & 68.1 & 4.2 \\
4008 & 355 & 95.0 & 10 & 64.1 & 39.2 & 11.6 & 73.6 & 4.5 \\
\multicolumn{8}{c}{ F-value of grain yields $=10.71 * * *, s_{x}=11.8 \%$} \\
Placement/broadcasting F-value $=27.97 * * *$
\end{tabular}

2148

$\begin{array}{lrrrrrr}100 & 0 & 72.0 & 37.0 & 9.1 & 51.1 & 3.5 \\ 138 & 2 & 72.7 & 41.1 & 12.3 & 50.9 & 3.7 \\ 221 & 7 & 72.6 & 41.3 & 10.2 & 66.2 & 4.3 \\ 213 & 6 & 73.7 & 42.5 & 12.5 & 59.9 & 3.9 \\ 269 & 25 & 72.0 & 42.4 & 12.4 & 73.7 & 4.4\end{array}$

F-value of grain yields $=57.35 * * *, s_{\bar{x}}=4.77 \%$

Placement/broadcasting F-value $=60.46$

\begin{tabular}{lccccccc}
2364 & 100 & 82.8 & 0 & 52.1 & 35.0 & 11.2 & 68.8 \\
3449 & 146 & 86.0 & 0 & 51.8 & 33.0 & 10.8 & 79.1 \\
3680 & 156 & 93.0 & 0 & 53.7 & 34.6 & 11.3 & 78.1 \\
3526 & 149 & 86.5 & 0 & 48.2 & 32.7 & 12.1 & 81.9 \\
3782 & 160 & 92.8 & 0 & 50.0 & 32.7 & 12.5 & 84.2 \\
\multicolumn{8}{c}{ F-value of grain yields $=8.67 * * *, s_{\mathbf{x}}=5.4 \%$}
\end{tabular}

Falling number

$\begin{array}{llrlll}2604 & 100 & 14 & 82.3 & 34.0 & 231 \\ 3455 & 133 & 3 & 83.7 & 36.6 & 238 \\ 4205 & 161 & 14 & 82.8 & 36.1 & 249 \\ 4042 & 155 & 10 & 82.8 & 36.8 & 224 \\ 4111 & 158 & 47 & 81.6 & 34.0 & 214\end{array}$

F-value of grain yields $=18.48 * * *, s_{\bar{x}}=5.8 \%$ Placement/broadcasting F-value $=8.34 *$ 


\begin{tabular}{|c|c|c|c|c|c|c|c|c|c|}
\hline Treatment & $\begin{array}{l}\text { Grair } \\
\frac{\text { g }}{\not 00}\end{array}$ & ield & 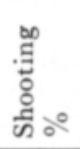 & 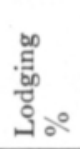 & 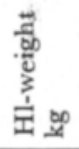 & $\begin{array}{l}\dot{3} \\
\text { io } \\
\delta_{0}^{\circ}\end{array}$ & $\begin{array}{l}00 \\
.5 \\
0 \\
0 \\
0\end{array}$ & 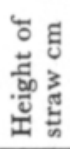 & 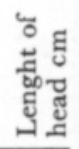 \\
\hline \multicolumn{10}{|l|}{ Apu-wheat 1968} \\
\hline 1. & 1354 & 100 & 57.8 & 2 & 73.2 & 31.0 & 13.8 & 319 & \\
\hline 2. See above Paavo-barley & 1858 & 137 & 72.5 & 10 & 73.8 & 31.8 & 13.8 & 311 & \\
\hline 3. $" \Rightarrow n$ & 2135 & 158 & 84.0 & 12 & 74.3 & 29.6 & 14.6 & 332 & \\
\hline 4. & 2247 & 166 & 80.0 & 12 & 73.3 & 28.8 & 15.4 & 272 & \\
\hline \multirow[t]{2}{*}{5.} & 2074 & 153 & 70.0 & 16 & 72.1 & 27.7 & 15.4 & 287 & \\
\hline & \multicolumn{9}{|c|}{$\begin{array}{l}\text { F-value of grain yields }=3.17 *, \mathrm{~s}_{\mathbf{x}}=10.1 \% \\
\text { Placement/broadcasting F-value }=0.15\end{array}$} \\
\hline
\end{tabular}

\title{
Svenno-wheat 1969
}

Met hod A

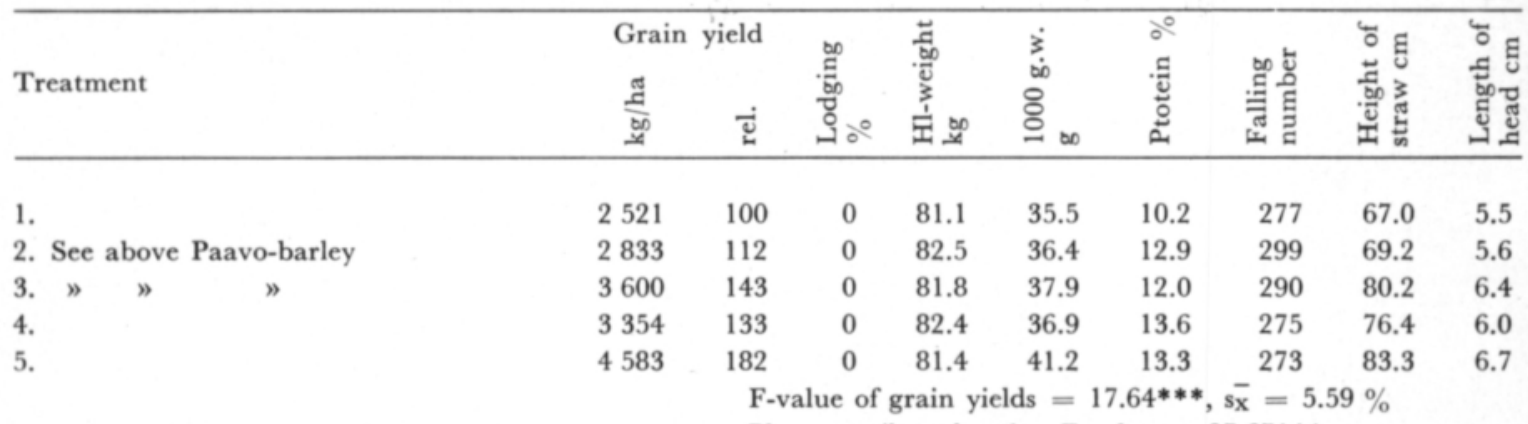

Met hod B

1.

2. See above Paavo-barley

3. " " "

4.

5.

\begin{tabular}{rllllllll}
2107 & 100 & 0 & 82.8 & 35.6 & 9.6 & 257 & 64.4 & 5.5 \\
2847 & 135 & 0 & 83.3 & 37.4 & 12.2 & 297 & 68.3 & 5.7 \\
3945 & 187 & 0 & 81.8 & 38.5 & 11.0 & 311 & 78.7 & 6.4 \\
3877 & 184 & 0 & 83.3 & 37.9 & 12.8 & 297 & 70.6 & 5.9 \\
4997 & 237 & 0 & 81.8 & 41.2 & 12.0 & 273 & 83.9 & 6.7 \\
\multicolumn{8}{c}{ F-value of grain yields $=64.96 * * *, s_{x}^{*}=3.9 \%$} \\
Placement/broadcasting F-value $=64.87 * * *$
\end{tabular}

\begin{abstract}
$A=$ Seed sown in line with the fertilizer rows
$\mathrm{B}=$ Seed sown across the fertilizer rows
\end{abstract}

Average value of grain yields of all the crops

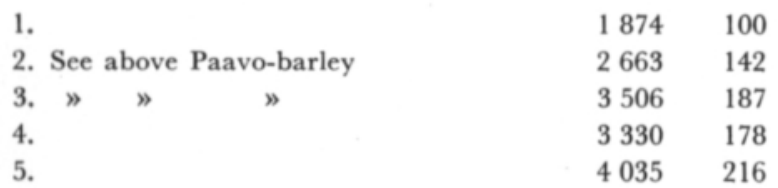


Table 5. Comparison tests with nitrochalk and urea in 1966-1969.

Wheat in 1966-69. Soil type sandy clay. Soil analysis in 1967: pH 5.9, Ca 2400, P 4.0 and K $190 \mathrm{mg} / \mathrm{l}$. Fertilization in spring: $800 \mathrm{~kg} / \mathrm{ha}$ of PK-fertilizer $(0-16.5-16.5), \mathrm{N}$ in different forms in $196675 \mathrm{~kg} / \mathrm{ha}$, in $1967137 \mathrm{~kg} / \mathrm{ha}$, in $196890 \mathrm{~kg} / \mathrm{ha}$ and in $196987 \mathrm{~kg} / \mathrm{ha}$. Test made with Svenno-wheat. Size of test plot $10 \times 120 \mathrm{~m}$. Harvested plot $6 \times 120 \mathrm{~m}, 2$ replications.

Barley in 1966-68. Soil type silty clay. Soil analysis in 1967: pH 5.8, Ca 2300, P 3.0 and K $215 \mathrm{mg} / \mathrm{l}$. Fertilization in spring: $800 \mathrm{~kg} / \mathrm{ha}$ of PK-fertilizer $(0-16.5-16.5), \mathrm{N}$ in different forms $100 \mathrm{~kg} / \mathrm{ha} / \mathrm{year}$. Test made with barley. Size of test plot $7.5 \times 65 \mathrm{~m}$. Harvested plot $6 \times 65 \mathrm{~m}, 2$ replications.

\begin{tabular}{|c|c|c|c|c|c|}
\hline \multirow{2}{*}{ Treatment } & Grain yield & \multirow{2}{*}{$\begin{array}{c}\text { Lodging } \\
\%\end{array}$} & \multirow{2}{*}{$\begin{array}{c}\text { Hl- } \\
\text { weight } \\
\text { kg }\end{array}$} & \multirow{2}{*}{$\begin{array}{c}1000 \text { g.w. } \\
\text { g }\end{array}$} & \multirow{2}{*}{$\begin{array}{c}\text { Protein } \\
\%\end{array}$} \\
\hline & $\mathrm{kg} / \mathrm{ha}$ & & & & \\
\hline
\end{tabular}

Test with wheat

Wheat 1966

$\begin{array}{lrrrrr}\text { 1. PK-fertilizing } & 1960 & 100 & 0 & 74.5 & 39.3 \\ \text { 2. In addition Nitrochalk, placement } & 2410 & 123 & 0 & 70.8 & 40.8 \\ \text { 3. " } & 1790 & 91 & 0 & 71.3 & 37.4\end{array}$

\section{Wheat 1967}

1.

2.

$\begin{array}{llrll}2160 & 100 & 0 & 82.9 & 36.1 \\ 3590 & 166 & 45 & 80.8 & 35.6 \\ 3490 & 160 & 35 & 82.6 & 36.7\end{array}$

\section{Wheat 1968}

1.

2.

3.

\section{Wheat 1969}

1.

2.

3.

Wheat 1969 2. test on sandy soil

1.

2.

3.

$\begin{array}{lll}1300 & 100 \\ 3250 & 250 \\ 3110 & 239 \\ & & \\ 1470 & \\ 2610 & 100 \\ 2190 & 178 \\ & & \end{array}$

$1980 \quad 100$

$3490 \quad 176$

$2880 \quad 145$

Fertilization $80 \mathrm{~kg} \mathrm{~N} / \mathrm{ha}$. Test plot $2.5 \times 30 \mathrm{~m} .3$ replications. 


\begin{tabular}{|c|c|c|c|c|c|c|}
\hline \multirow{2}{*}{ Treatment } & \multicolumn{2}{|c|}{ Grain yield } & \multirow{2}{*}{$\begin{array}{c}\text { Lodging } \\
\%\end{array}$} & \multirow{2}{*}{$\begin{array}{c}\text { Hl- } \\
\text { weight } \\
\text { kg }\end{array}$} & \multirow{2}{*}{$\begin{array}{c}1000 \text { g.w. } \\
\text { g }\end{array}$} & \multirow{2}{*}{$\begin{array}{c}\text { Protein } \\
\%\end{array}$} \\
\hline & $\mathrm{kg} / \mathrm{ha}$ & rel. & & & & \\
\hline
\end{tabular}

Tests with wheat, on an average:

(in 1966-1969) (5 tests)

$\begin{array}{llll}\text { 1. } & 1770 & 100 \\ \text { 2. } & 3070 & 173 & \\ \text { 3. } & 2690 & 152 & \\ & & \text { Treatment } 2 / 3 \quad \mathrm{t}=4.22 * *\end{array}$

\section{Tests with barley}

1. Control yield

2. Nitrochalk, in rows

3. Urea in rows

4. Nitrochalk, broadcasting

$\begin{array}{rllll}300 & 100 & 0 & 63.8 & 41.8 \\ 2000 & 667 & 0 & 62.9 & 43.3 \\ 1060 & 333 & 0 & 63.1 & 41.8 \\ 1510 & 503 & 0 & 61.3 & 39.5\end{array}$

Barley 1967

1.

2.

3.

4.

$\begin{array}{llrll}1520 & 100 & 0 & 69.5 & 38.6 \\ 2670 & 176 & 13 & 67.8 & 42.5 \\ 2320 & 153 & 5 & 68.1 & 41.8 \\ 2400 & 158 & 5 & 68.1 & 41.9\end{array}$

\section{Barley 1968}

1.

2.

3.

4.

\begin{tabular}{lrrrrr}
1110 & 100 & 0 & 55.6 & 33.5 & 11.5 \\
2830 & 255 & 13 & 57.0 & 33.2 & 10.4 \\
3010 & 274 & 10 & 57.3 & 32.1 & 11.2 \\
2980 & 268 & 9 & 55.6 & 33.0 & 11.1 \\
\hline
\end{tabular}

Tests with barley, average:

(3 tests)

$$
1 .
$$

2.

3.

4.

$\begin{array}{rr}980 & 100 \\ 2500 & 255 \\ 2130 & 217 \\ 2300 & 235\end{array}$

Treatment $2 / 3 \quad \mathrm{t}=1.75(\mathrm{t} 5 \%=2.57)$

Tests with barley and wheat, average:

$\begin{array}{lll}1 . & 1380 & 100 \\ 2 . & 2790 & 202 \\ 3 . & 2410 & 175\end{array}$


Table 6. Comparison of nitrochalk and urea in potassium metaphosphate tests in 1966-1969.

Soil type silty sandy clay. Soil analysis in $1967 \mathrm{pH} \mathrm{5.6,} \mathrm{Ca} \mathrm{2300,} \mathrm{P} 4.0$ and K $235 \mathrm{mg} / \mathrm{l}$. Test made with Svenno-wheat in 1966-67, with Otra-barley in 1968. Size of test plot $8 \times 30 \mathrm{~m}$, harvested plot $6 \times 30 \mathrm{~m} .3-4$ replications. Nutrient amounts correspond to $500 \mathrm{~kg} / \mathrm{ha}$ of fertilizer $15-20-15$ $\left(\mathrm{N}-\mathrm{P}_{2} \mathrm{O}_{5}-\mathrm{K}_{2} \mathrm{O}\right)$. Straight fertilizers: superphosphate (Psf), potassium chloride, potassium metaphosphate, nitrochalk and urea. Potassium metaphosphate: $\mathrm{P}_{2} \mathrm{O}_{5} 57 \%$ and $\mathrm{K}_{5} \mathrm{O} 36 \%$. The fertilizers were broadcast.

\begin{tabular}{|c|c|c|c|c|c|c|c|}
\hline \multirow[b]{2}{*}{ Treatment } & \multicolumn{2}{|c|}{ Grain yield } & \multirow{2}{*}{ 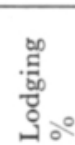 } & \multirow{2}{*}{ 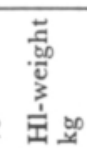 } & \multirow{2}{*}{ 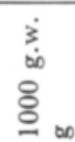 } & \multirow{2}{*}{ 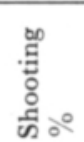 } & \multirow{2}{*}{ 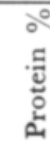 } \\
\hline & $\frac{\pi}{80}$ & $\overline{\mathrm{D}}$ & & & & & \\
\hline
\end{tabular}

\section{Svenno-wheat (1966-67)}

$\begin{array}{lllllll}\text { 1. Control yield } & 1992 & 100 & 0 & 82.1 & 35.2\end{array}$

2. Superphosph. + potassium chloride + nitrochalk $\quad \begin{array}{llllll}3195 & 160 & 0 & 82.6 & 37.0\end{array}$

3. Potassium metaphosphate + nitrochalk $\quad \begin{array}{llllll}3219 & 162 & 0 & 82.3 & 37.3\end{array}$

4. " " + urea $\quad 3162 \quad 159 \quad 0032.1 \quad 37.5$

F-value of grain yields in $1966=10.35$, $\overline{\mathbf{x}}=4.2 \%$

$\mathrm{F}$-value of grain yields in $1967=14.25$

$\mathbf{s} \bar{x}$

Otra-barley (1968)

1. Control yield

$\begin{array}{lllllll}793 & 100 & 0 & 62.1 & 42.4 & 73.8 & 9.80\end{array}$

2. Superphosph. + potassium chloride + nitrochalk $\begin{array}{llllllll}2 & 235 & 281 & 0 & 60.2 & 33.5 & 77.5 & 10.15\end{array}$

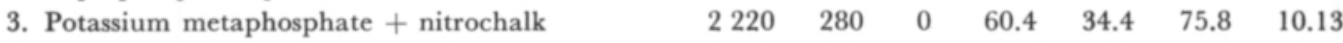

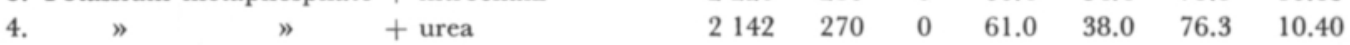

Winter wheat, Linna (1969)

$\begin{array}{lll}\text { 1. Control yield } & 4372 \quad 100\end{array}$

2. Superphosph. + potassium chloride + nitrochalk $6012 \quad 138$

3. Potassium metaphosphate + nitrochalk $\quad 5514 \quad 126$

4. " " + urea $\quad 5305121$

F-value of grain yields $=9.73, \mathrm{~s}_{\overline{\mathrm{x}}}=4.16 \%$

Average (test years 1966-69)

$\begin{array}{lll}\text { 1. Control yield } & 2287 & 100\end{array}$

2. Superphosph. + potassium chloride + nitrochalk $3659 \quad 160$

3. Potassium metaphosphate + nitrochalk $\quad 3543 \quad 155$

4. " $"$ + urea 3443150

Statistically reliable differences between grain yields were not observed in connection with the tested crops in treatments $2-4$. 